\title{
Flavor ratios of astrophysical neutrinos interacting with stochastic gravitational waves having arbitrary spectra
}

\author{
Maxim Dvornikov \\ Pushkov Institute of Terrestrial Magnetism, Ionosphere and Radiowave Propagation (IZMI- \\ RAN), 108840 Troitsk, Moscow, Russia \\ E-mail: maxdvo@izmiran.ru
}

\begin{abstract}
We study the evolution and oscillations of fixed massive neutrinos interacting with stochastic gravitational waves (GWs). The energy spectrum of these GWs is Gaussian, with the correlator of the amplitudes being arbitrary. We derive the equation for the density matrix for flavor neutrinos in this case. In the two flavors approximation, this equation can be solved analytically. We find the numerical solution for the density matrix in the general case of three neutrino flavors. We consider merging binary black holes as sources of stochastic GWs with realistic spectra. Both normal and inverted mass orderings are analyzed. We discuss the relaxation of the neutrino fluxes in stochastic GWs emitted mainly by supermassive black holes. In this situation, we obtain the range of energies and the propagation lengths for which the relaxation process is the most efficient. We discuss the application of our results for the observation of fluxes of astrophysical neutrinos.
\end{abstract}

Keywords: neutrino astronomy, neutrino detectors, gravitational waves/sources 


\section{Contents}

1 Introduction 1

2 Density matrix evolution accounting for an arbitrary spectrum of GWs 2

3 Astrophysical applications $\quad 5$

4 Discussion $\quad 8$

A Fluxes of neutrinos emitted by random sources $\quad 10$

\section{Introduction}

Flavor transformations of a neutrino beam, called neutrino flavor oscillations, recently confirmed experimentally (see, e.g. ref. [1]), are the direct indication to the nonzero masses of these particles and the mixing between different neutrino states. Various external fields are known to contribute, or even enhance, neutrino oscillations [2]. The gravitational interaction, in spite of its weakness, is supposed to modify the neutrino oscillations process.

Neutrino oscillations in gravitational fields were first considered in ref. [3] and, subsequently, in multiple papers. Many of them are reviewed in ref. [4]. A gravitational field is supposed to modify the phase of a massive neutrino wave function [5]. This phase obeys the Hamilton-Jacobi equation, which should be written down in curved spacetime.

It is important to study neutrino oscillations in gravitational fields considering not only static fields, but also time dependent gravitational backgrounds like a gravitational wave (GW). It is inspired by the recent GWs detection reported in ref. [6]. Now multiple sources of GWs, mainly as coalescing binary compact objects, are catagolized in ref. [7]. Sources of GWs are expected to emit significant neutrino fluxes [8]. Thus, emitted GWs can influence the propagation and oscillations of astrophysical neutrinos. There are active searches of neutrinos emitted by merging binaries, which are the sources of GWs [9].

We studied neutrino flavor and spin (i.e., transitions between active left and sterile right states) oscillations under the influence of gravitational fields in refs. [10-14]. Neutrino spin oscillations in GWs were considered in ref. [13]. The evolution of the spin of a fermion in GW was discussed in ref. [15]. The action of GWs on the propagation and flavor oscillations of astrophysical neutrinos was studied in refs. [14, 16].

In the present work, we continue our research in ref. [14]. We study the evolution of a beam of fixed massive neutrinos interacting with stochastic GWs with an arbitrary energy spectrum. In section 2, we derive the equation for the density matrix for flavor neutrinos and solve it analytically in the two flavors approximation. The numerical solution of this equation for the general situation of the three neutrino flavors is obtained in section 3, where we study astrophysical applications. We consider coalescing binary BHs as sources of stochastic GWs. We obtain the maximal energy and the minimal propagation length, at which the fluxes of flavor neutrinos reach their asymptotic values. We discuss our results and consider their implication for the observation of astrophysical neutrinos in section 4. The averaged fluxes of neutrinos involved in flavor oscillations in vacuum are obtained in appendix A. 


\section{Density matrix evolution accounting for an arbitrary spectrum of GWs}

We study the system of three flavor neutrinos $\left(\nu_{e}, \nu_{\mu}, \nu_{\tau}\right)$ with nonzero mixing. One can diagonalize the mass matrix of such neutrinos using the neutrino mass eigenstates $\psi_{a}, a=$ $1,2,3$, which have the definite masses $m_{a}$. Flavor and mass eigenstates are related by the matrix transformation,

$$
\nu=U \psi,
$$

where $U$ is the mixing matrix.

In the most general situation of three neutrino flavors $\nu=\left(\nu_{e}, \nu_{\mu}, \nu_{\tau}\right)$, the mixing matrix in eq. (2.1) can be parameterized in the following form [17, pgs. 111-116]:

$$
U=\left(\begin{array}{ccc}
1 & 0 & 0 \\
0 & c_{23} & s_{23} \\
0 & -s_{23} & c_{23}
\end{array}\right) \cdot\left(\begin{array}{ccc}
c_{13} & 0 & s_{13} e^{-\mathrm{i} \delta_{\mathrm{CP}}} \\
0 & 1 & 0 \\
-s_{13} e^{\mathrm{i} \delta_{\mathrm{CP}}} & 0 & c_{13}
\end{array}\right) \cdot\left(\begin{array}{ccc}
c_{12} & s_{12} & 0 \\
-s_{12} & c_{12} & 0 \\
0 & 0 & 1
\end{array}\right)
$$

where $c_{a b}=\cos \theta_{a b}, s_{a b}=\sin \theta_{a b}, \theta_{a b}$ are the corresponding vacuum mixing angles, and $\delta_{\mathrm{CP}}$ is the $\mathrm{CP}$ violating phase. The values of these parameters can be found in ref. [18]. The mixing matrix takes more simple form in the frequently used two flavors approximation,

$$
U=\left(\begin{array}{cc}
\cos \theta & \sin \theta \\
-\sin \theta & \cos \theta
\end{array}\right)
$$

where $\theta$ is the only mixing angle.

We suppose that these flavor neutrinos move in curved spacetime with background gravitational field in the form of a plane gravitational wave with the circular polarization propagating along the $z$-axis. The interval in this case has the form [19],

$$
\mathrm{d} s^{2}=g_{\mu \nu} \mathrm{d} x^{\mu} \mathrm{d} x^{\nu}=\mathrm{d} t^{2}-(1-h \cos \phi) \mathrm{d} x^{2}-(1+h \cos \phi) \mathrm{d} y^{2}+2 \mathrm{~d} x \mathrm{~d} y h \sin \phi-\mathrm{d} z^{2},
$$

where $h$ is the dimensionless amplitude of the wave, $\phi=(\omega t-k z)$ is the phase of the wave, $\omega$ is frequency of the wave, and $k$ is the wave vector. In eq. (2.4), we use the Cartesian coordinates $x^{\mu}=(t, x, y, z)$.

The dynamics of flavor oscillations $\nu_{\alpha} \leftrightarrow \nu_{\beta}$ is described by the effective Schrodinger equation,

$$
\mathrm{i} \dot{\nu}=H_{f} \nu, \quad H_{f}=U H_{m} U^{\dagger}, \quad H_{m}=H_{m}^{(\mathrm{vac})}+H_{m}^{(g)},
$$

where $H_{f}$ and $H_{m}$ are the effective Hamiltonians in the flavor and mass bases, $H_{m}^{(\mathrm{vac})}=$ $\frac{1}{2 E} \operatorname{diag}\left(m_{1}^{2}, m_{2}^{2}, m_{3}^{2}\right)$ is the part of the Hamiltonian responsible for vacuum oscillations, $E \approx p$ is the mean neutrino energy, $p$ is the momentum of mass eigenstates.

Using the results of ref. [20], we have derived in ref. [14] the contribution to $H_{m}$ from the neutrino interaction with GW. We assume that

$$
\omega L\left|\beta_{a}-\beta_{b}\right| \ll 1, \quad a, b=1, \ldots, 3,
$$

where $L$ is the neutrino propagation distance, $\beta_{a}=p / E_{a}$ is the velocity of the mass eigenstate, $p$ is the mean neutrino momentum, and $E_{a}=\sqrt{m_{a}^{2}+p^{2}}$ is the energy of the mass eigenstate. In this case, $H_{m}^{(g)}$ have only diagonal components which have the form,

$$
\left(H_{m}^{(g)}\right)_{a a}=-\frac{p^{2} h}{2 E_{a}} \sin ^{2} \vartheta \cos 2 \varphi \approx-h A\left(p-\frac{m_{a}^{2}}{2 p}\right) \rightarrow h A \frac{m_{a}^{2}}{2 E},
$$


where $\vartheta$ and $\varphi$ are the spherical angles fixing the neutrino momentum with respect to the wave vector $\mathbf{k}=(0,0, k)$ of $\mathrm{GW}$ and $A(\vartheta, \varphi)=\frac{1}{2} \sin ^{2} \vartheta \cos 2 \varphi$. In eq. (2.7), we keep only the linear term in $h$.

In eq. (2.7), we assume that the momenta of all mass eigenstates are equal to $p$. Then, accounting for the fact that neutrinos are ultrarelativistic, i.e. $p \gg m_{a}$, we expand $H_{m}^{(g)}$ in the small parameters $m_{a}^{2} / p^{2}$ and omit the term which is equal for all mass eigenstates. It is known that such a term does not contribute to the dynamics of neutrino oscillations. Eventually, we replace $p \rightarrow E$ in the final expression. The same technique is used to derive the expression for $H_{m}^{(\text {vac })}$ given above.

Finally, using eqs. (2.5) and (2.7), we get the total effective Hamiltonian for flavor eigenstates in the form,

$$
H_{f}=H_{0}+H_{1}, \quad H_{0}=U H_{m}^{(\mathrm{vac})} U^{\dagger}, \quad H_{1}=\xi H_{0},
$$

where $\xi=h A$. Note that $H_{0}$ in eq. (2.8) is the constant matrix.

Now we suppose that neutrinos interact with stochastic GWs, i.e. the angles $\vartheta$ and $\varphi$, as well as $h$ are random functions of time. In this case, it is convenient to study the evolution of the density matrix $\rho$ rather than the wave function $\nu$. In this approach, the diagonal elements of $\rho$ are the probabilities to detect a certain flavor in a neutrino beam. Using the results of ref. [21], we get the equation for $\rho_{\mathrm{I}}(t)=\exp \left(\mathrm{i} H_{0} t\right) \rho(t) \exp \left(-\mathrm{i} H_{0} t\right)$ in the form,

$$
\mathrm{i} \dot{\rho}_{\mathrm{I}}=\left[H_{\mathrm{I}}, \rho_{\mathrm{I}}\right],
$$

where $H_{\mathrm{I}}=\exp \left(\mathrm{i} H_{0} t\right) H_{1} \exp \left(-\mathrm{i} H_{0} t\right)=H_{1}$.

The replacement of the density matrix $\rho \rightarrow \rho_{\mathrm{I}}$ means the consideration of the interaction picture [22] for the neutrino flavor eigenstates. It is used frequently in quantum mechanics to develop a perturbative approach (see, e.g., ref. [21]).

The formal solution of eq. (2.9) can be represented in the form of a series, which should be averaged over a certain time interval. We suppose that the amplitudes of GW form the Gaussian stochastic process. Thus, only even terms in this series survive since all odd correlators, like $\left\langle h\left(t_{1}\right) h\left(t_{2}\right) h\left(t_{3}\right)\right\rangle$ etc, are vanishing. Eventually one has

$$
\begin{aligned}
\left\langle\rho_{\mathrm{I}}\right\rangle(t)= & \rho_{0}-\left\langle A^{2}\right\rangle\left[H_{0},\left[H_{0}, \rho_{0}\right]\right] \int_{0}^{t} \mathrm{~d} t_{1} \int_{0}^{t_{1}} \mathrm{~d} t_{2} f\left(\left|t_{1}-t_{2}\right|\right) \\
& +\left\langle A^{2}\right\rangle^{2}\left[H_{0},\left[H_{0},\left[H_{0},\left[H_{0}, \rho_{0}\right]\right] \int_{0}^{t} \mathrm{~d} t_{1} \int_{0}^{t_{1}} \mathrm{~d} t_{2} \int_{0}^{t_{2}} \mathrm{~d} t_{3} \int_{0}^{t_{3}} \mathrm{~d} t_{4}\right.\right. \\
& \times\left[f\left(\left|t_{1}-t_{2}\right|\right) f\left(\left|t_{3}-t_{4}\right|\right)+f\left(\left|t_{1}-t_{3}\right|\right) f\left(\left|t_{2}-t_{4}\right|\right)\right. \\
& \left.+f\left(\left|t_{1}-t_{4}\right|\right) f\left(\left|t_{2}-t_{3}\right|\right)\right]-\ldots,
\end{aligned}
$$

where $\rho_{0}=\rho_{\mathrm{I}}(0)=\rho(0)$ is the initial density matrix, $f\left(\left|t_{1}-t_{2}\right|\right)=\left\langle h\left(t_{1}\right) h\left(t_{2}\right)\right\rangle$ is the correlator of GW amplitudes, and

$$
\left\langle A^{2}\right\rangle=\frac{1}{2 \pi^{2}} \int_{0}^{\pi} \mathrm{d} \vartheta \int_{0}^{2 \pi} \mathrm{d} \varphi A^{2}(\vartheta, \varphi)=\frac{3}{64}
$$

is the mean value of the angle factor squared.

To derive eq.(2.10) we assume that both $\vartheta$ and $\varphi$ have the $\delta$-correlated Gaussian distributions, which is a reasonable assumption since stochastic GWs intersect a neutrino trajectory 
randomly. However, unlike ref. [14], we do not assume that the amplitude of GW has the same distribution, i.e. $f\left(\left|t_{1}-t_{2}\right|\right) \nsim \delta\left(t_{1}-t_{2}\right)$ is the arbitrary function.

After lengthy but straightforward calculations, we transform eq. (2.10) to the form,

$$
\left\langle\rho_{\mathrm{I}}\right\rangle(t)=\rho_{0}-a\left\langle A^{2}\right\rangle\left[H_{0},\left[H_{0}, \rho_{0}\right]\right]+\frac{1}{2 !}\left(a\left\langle A^{2}\right\rangle\right)^{2}\left[H_{0},\left[H_{0},\left[H_{0},\left[H_{0}, \rho_{0}\right]\right]-\ldots,\right.\right.
$$

where

$$
a(t)=\int_{0}^{t} \mathrm{~d} t_{1} \int_{0}^{t_{1}} \mathrm{~d} t_{2} f\left(\left|t_{1}-t_{2}\right|\right)=\int_{0}^{t} \mathrm{~d} t_{1}\left(t-t_{1}\right) f\left(\left|t_{1}\right|\right) .
$$

One can check that eq. (2.12) is the formal solution of the following equation:

$$
\frac{\mathrm{d}}{\mathrm{d} t}\left\langle\rho_{\mathrm{I}}\right\rangle(t)=-g(t)\left\langle A^{2}\right\rangle\left[H_{0},\left[H_{0},\left\langle\rho_{\mathrm{I}}\right\rangle(t)\right]\right],
$$

where

$$
g(t)=\int_{0}^{t} \mathrm{~d} t_{1} f\left(\left|t-t_{1}\right|\right),
$$

which is the generalization of the results of ref. [14] for the arbitrary correlator $f\left(\left|t_{1}-t_{2}\right|\right)$ of the amplitudes of GWs. If we choose $f\left(\left|t_{1}-t_{2}\right|\right)=2 \tau\left\langle h^{2}\right\rangle \delta\left(t_{1}-t_{2}\right)$, where $\tau$ is the phenomenological correlation time, and use eqs. (2.11) and (2.15), then eq. (2.14) reproduces eq. (2.17) in ref. [14].

Let us study the two flavors approximation. In this case,

$$
H_{0}=\frac{\Delta m_{21}^{2}}{4 E}(\boldsymbol{\sigma} \mathbf{n}), \quad \mathbf{n}=(\sin 2 \theta, 0,-\cos 2 \theta),
$$

where $\Delta m_{21}^{2}=m_{2}^{2}-m_{1}^{2}$ and $\boldsymbol{\sigma}$ are the Pauli matrices. We can sum analytically the series in eq. (2.12). Indeed,

$$
\begin{aligned}
\left\langle\rho_{\mathrm{I}}\right\rangle(t)= & \rho_{0}-\left[\rho_{0}-(\boldsymbol{\sigma} \mathbf{n}) \rho_{0}(\boldsymbol{\sigma} \mathbf{n})\right] \frac{\lambda}{2}\left(1-\frac{\lambda}{2 !}+\frac{\lambda^{2}}{3 !}-\ldots\right) \\
& =\frac{1}{2}\left[\rho_{0}\left(1+e^{-\lambda}\right)+(\boldsymbol{\sigma} \mathbf{n}) \rho_{0}(\boldsymbol{\sigma} \mathbf{n})\left(1-e^{-\lambda}\right)\right],
\end{aligned}
$$

where $\lambda=a\left\langle A^{2}\right\rangle\left(\Delta m_{21}^{2}\right)^{2} / 4 E^{2}$. If we choose the $\delta$-correlated Gaussian distribution, then $a=\tau t\left\langle h^{2}\right\rangle$ and eq. (2.17) reproduces the corresponding result of ref. [14].

The correlation function $\langle h(t) h(0)\rangle=f(|t|)$ can be expressed in terms of the spectral density $S(f)$ as $[23]$

$$
\langle h(t) h(0)\rangle=\int_{0}^{\infty} \mathrm{d} f \cos (2 \pi f t) S(f),
$$

where $f$ is the frequency measured in Hz. Instead of the spectral density in eq. (2.18) it is convenient to consider the energy density of stochastic GWs, $\Omega(f)$, per logarithmic frequency interval with respect to the closure density of the universe $\rho_{c}=\frac{3 \mathrm{H}_{0}^{2}}{8 \pi G}=0.53 \times 10^{-5} \mathrm{GeV}$. $\mathrm{cm}^{-3}[24]$,

$$
S(f)=\frac{8 G \rho_{c}}{\pi f^{3}} \Omega(f),
$$

where $\mathrm{H}_{0}$ is the Hubble constant and $G=6.9 \times 10^{-39} \mathrm{GeV}^{-2}$ is the Newton constant. The function $g(t)$ in eq. (2.15) takes the form,

$$
g(t)=\frac{4 G \rho_{c}}{\pi^{2}} \int_{0}^{\infty} \frac{\mathrm{d} f}{f^{4}} \sin (2 \pi f t) \Omega(f),
$$

which should be used in the differential eq. (2.14). 


\section{$3 \quad$ Astrophysical applications}

In this section, we study flavor transformations of a neutrino beam under the influence of a realistic stochastic GW background. This problem was studied previously in ref. [14], where coalescing black holes (BHs) were considered. However, the correlator of GW amplitudes was assumed in ref. [14] to be of the form, $\left\langle h\left(t_{1}\right) h\left(t_{2}\right)\right\rangle \sim \delta\left(t_{1}-t_{2}\right)$. Spectra of realistic stochastic GW backgrounds were mentioned in ref. [27] to be approximated by power laws, $\Omega(f) \sim f^{\alpha}$, with the frequency $f$ being in a confined region. Thus, the approximation made in ref. [14] is quite rough.

We should start with the specification of the initial condition for eq. $(2.14), \rho_{\mathrm{I}}(0)=\rho(0)$. We study the evolution of astrophysical neutrinos created in decays of charged pions [25]. In this case, one has that the fluxes of flavor neutrinos at a source are $\left(F_{\nu_{e}}: F_{\nu_{\mu}}: F_{\nu_{\tau}}\right)_{\mathrm{S}}=(1$ : $2: 0)$. Thus $\rho_{\mathrm{I}}(0)=\operatorname{diag}(1 / 3,2 / 3,0)$.

First, we study the GW background from coalescing supermassive BHs (SMBHs). In this case, we can approximate $\Omega(f)$ by $[26]$

$$
\Omega(f)= \begin{cases}\Omega_{0}, & \text { if } f_{\min }<f<f_{\max }, \\ 0, & \text { otherwise }\end{cases}
$$

where $\Omega_{0} \sim 10^{-9}, f_{\min } \sim 10^{-10} \mathrm{~Hz}$, and $f_{\max } \sim 10^{-1} \mathrm{~Hz}$. Note that this value of $\Omega_{0}$ does not violate the constraint established in ref. [27]. Using eq. (3.1), we get $g(t)$ in eq. (2.20) in the explicit form,

$$
\begin{aligned}
g(t)= & -\frac{2 G \rho_{c} \Omega_{0}}{3 \pi^{2}}\left\{\sin \left(2 \pi f_{\max } t\right) \frac{2-\left(2 \pi f_{\max } t\right)^{2}}{f_{\max }^{3}}-\sin \left(2 \pi f_{\min } t\right) \frac{2-\left(2 \pi f_{\min } t\right)^{2}}{f_{\min }^{3}}\right. \\
& +2 \pi t\left[\frac{\cos \left(2 \pi f_{\max } t\right)}{f_{\max }^{2}}-\frac{\cos \left(2 \pi f_{\min } t\right)}{f_{\min }^{2}}\right] \\
& \left.+(2 \pi t)^{3}\left[\operatorname{Ci}\left(2 \pi f_{\max } t\right)-\operatorname{Ci}\left(2 \pi f_{\min } t\right)\right]\right\},
\end{aligned}
$$

where $\operatorname{Ci}(x)=\gamma+\ln x+\int_{0}^{x} \frac{\cos t-1}{t} \mathrm{~d} t$ is the cosine integral and $\gamma \approx 0.577$.

In figures 1 and 2, we show the numerical solution of eqs. (2.14) and (3.2) with $\left\langle A^{2}\right\rangle=$ $\frac{3}{64}, \Omega_{0}$, and $f_{\text {min,max }}$ given above. The neutrino energy is in the range $E=\left(10^{2}-10^{4}\right) \mathrm{MeV}$ and the propagation distance is $L=1 \mathrm{kpc}$. We study the cases of both the normal and inverted orderings of the neutrino masses. The mass squared differences and the mixing angles are taken from ref. [18].

One can see in figures 1(b) and 2(b) that the contribution of GWs to neutrino oscillations is negligible at high neutrino energies since $H_{m}^{(g)} \sim E^{-1}$ in eq. (2.7). Indeed, one gets that $\left\langle\rho_{k k}^{(\mathrm{I})}\right\rangle(t)$ practically coincides with $\left\langle\rho_{k k}^{(\mathrm{I})}\right\rangle(0)=\operatorname{diag}(1 / 3,2 / 3,0)$ at $E=10 \mathrm{GeV}$.

Comparing the results in figures 1 (a) and 2(a) with the findings of ref. [14], where analogous problem was studied, we establish the more significant effect of GWs on the relaxation of the density matrix now. We find that $\left\langle\rho_{\mathrm{I}}\right\rangle$ reaches its asymptotic values at shorter $L$ and greater $E$. This discrepancy can be explained by the underestimation of the relaxation time $\tau$ in ref. [14]. In the present work, we take into account the spectrum of stochastic GWs exactly. Thus there is no need to approximate the correlator by a $\delta$-function.

The elements of $\left\langle\rho_{\mathrm{I}}\right\rangle$ are not the measurable quantities. The fluxes of flavor neutrinos $F_{\nu_{\lambda}}$ are proportional to the diagonal elements of the total density matrix $\langle\rho\rangle(t)=$ 


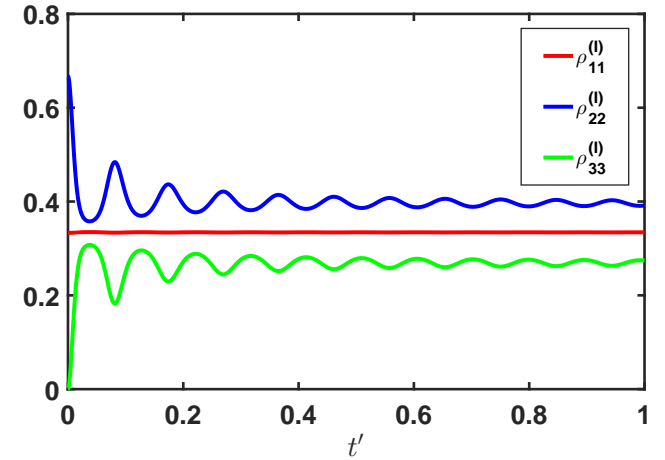

(a)

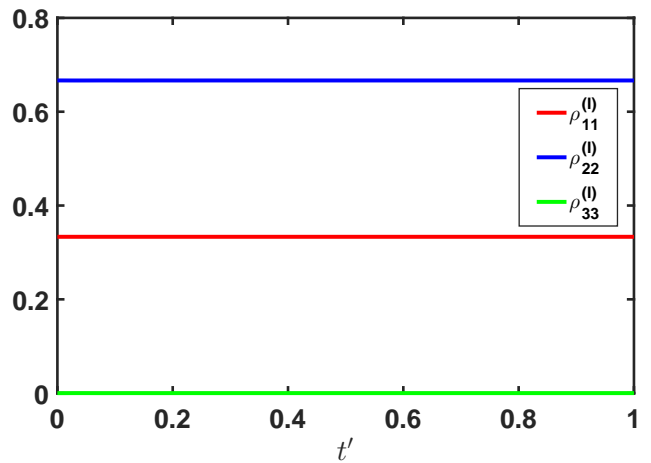

(b)

Figure 1. The diagonal elements of $\left\langle\rho_{\mathrm{I}}\right\rangle$ based on the numerical solution of eq. (2.14) versus $t^{\prime}=t / L$. Here we adopt the normal mass ordering with $\Delta m_{21}^{2}=7.5 \times 10^{-5} \mathrm{eV}^{2}, \Delta m_{31}^{2}=2.56 \times 10^{-3} \mathrm{eV}^{2}$, $\theta_{12}=0.6, \theta_{23}=0.85, \theta_{13}=0.15$, and $\delta_{\mathrm{CP}}=3.77$ (see ref. [18]). The neutrino beam propagation distance is $L=1 \mathrm{kpc}$. (a) $E=10^{2} \mathrm{MeV}$; and (b) $E=10 \mathrm{GeV}$.

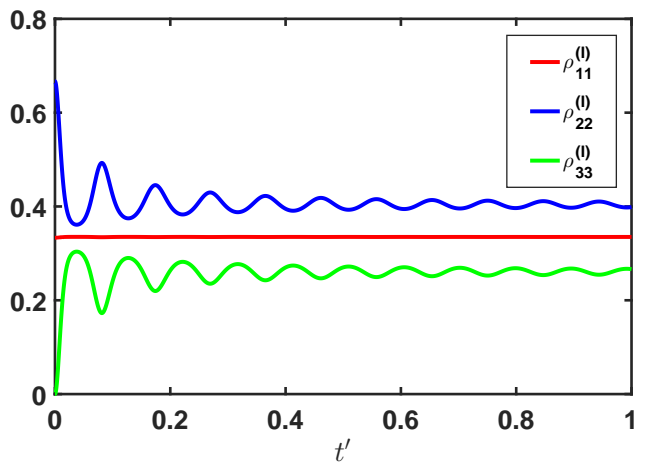

(a)

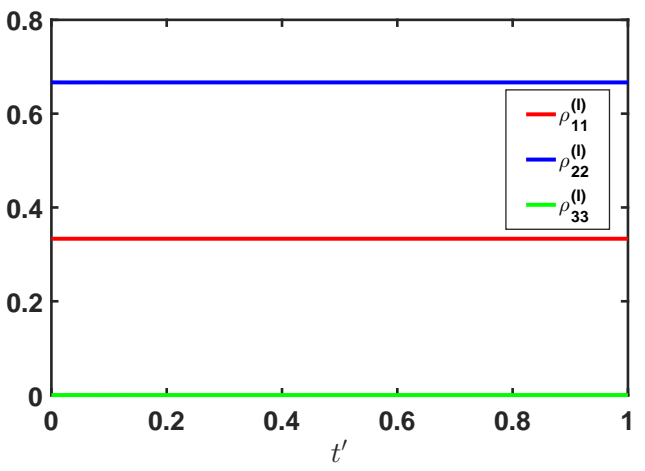

(b)

Figure 2. The same as in figure 1, but for the inverted mass ordering with $\Delta m_{21}^{2}=7.5 \times 10^{-5} \mathrm{eV}^{2}$, $\Delta m_{31}^{2}=-2.46 \times 10^{-3} \mathrm{eV}^{2}, \theta_{12}=0.59, \theta_{23}=0.85, \theta_{13}=0.15$, and $\delta_{\mathrm{CP}}=4.84$. (a) $E=10^{2} \mathrm{MeV}$; and (b) $E=10 \mathrm{GeV}$.

$\exp \left(-\mathrm{i} H_{0} t\right)\langle\rho\rangle_{\mathrm{I}}(t) \exp \left(\mathrm{i} H_{0} t\right)$. In figure 3 , we show $F_{\nu_{\lambda}}$ for $L=1 \mathrm{kpc}$ and $E=10^{2} \mathrm{MeV}$. We have demonstrated in figures $1(\mathrm{~b})$ and $2(\mathrm{~b})$ that GWs does not contribute to the evolution of the density matrix at $E=10 \mathrm{GeV}$. Thus we do not show the fluxes for $E=10 \mathrm{GeV}$.

It is difficult to distinguish the fluxes for different flavors in figure 3 by sight because of the rapid vacuum oscillations. However, if one averages the signal over the length of these oscillations, one gets the fluxes in a detector $\left\langle F_{\nu_{\lambda}}\right\rangle_{\oplus}$ which turn out to be different for different flavors. This additional averaging is equivalent to the situation when we study not only the action of stochastic GWs on the neutrino beam, but also consider randomly distributed neutrino sources.

We write down the values of $\left\langle F_{\nu_{\lambda}}\right\rangle_{\oplus}$ for $E=10 \mathrm{MeV}$ and $L=1 \mathrm{kpc}$ in the upper rows in tables 1 and 2 for the normal and inverted mass orderings. Firstly, one can see that the cases of the normal and inverted mass orderings are distinguishable. Secondly, $\left\langle F_{\nu_{\lambda}}\right\rangle_{\oplus}$ are not equal for different neutrino flavors. This feature is discussed in appendix A. We remind 


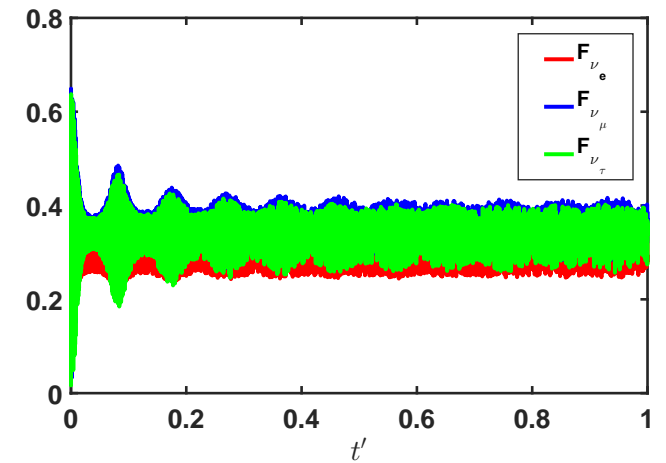

(a)

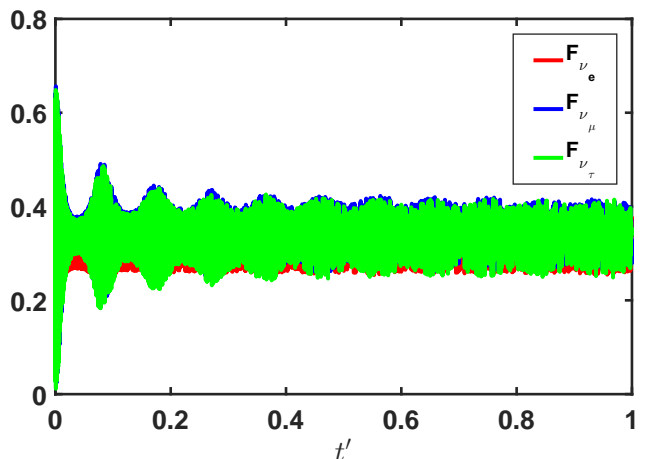

(b)

Figure 3. Fluxes of flavor neutrinos based on the numerical solution of eq. (2.14) with $g(t)$ in eq. (3.2) for $E=10^{2} \mathrm{MeV}$ and $L=1 \mathrm{kpc}$. (a) Normal mass ordering; and (b) inverted mass ordering.

\begin{tabular}{|l|l|l|l|}
\hline & $\left\langle F_{\nu_{e}}\right\rangle_{\oplus}$ & $\left\langle F_{\nu_{\mu}}\right\rangle_{\oplus}$ & $\left\langle F_{\nu_{\tau}}\right\rangle_{\oplus}$ \\
\hline \hline $\begin{array}{l}\text { Accounting for stochastic GWs and aver- } \\
\text { aging over the source position }\end{array}$ & 0.3038 & 0.3551 & 0.3411 \\
\hline Only averaging over the source position & 0.3029 & 0.3552 & 0.3419 \\
\hline $\begin{array}{l}\text { Relative contribution of stochastic GWs } \\
\text { (absolute value, in \%) }\end{array}$ & 0.3 & 0.03 & 0.2 \\
\hline
\end{tabular}

Table 1. Averaged fluxes of flavor neutrinos in a detector for the normal mass ordering for $E=$ $10^{2} \mathrm{MeV}$. The upper row is based on figure 3(a). The middle row corresponds to eq. (A.4). The lower row represents the absolute value of the relative contribution of stochastic GWs.

\begin{tabular}{|l|l|l|l|}
\hline & $\left\langle F_{\nu_{e}}\right\rangle_{\oplus}$ & $\left\langle F_{\nu_{\mu}}\right\rangle_{\oplus}$ & $\left\langle F_{\nu_{\tau}}\right\rangle_{\oplus}$ \\
\hline \hline $\begin{array}{l}\text { Accounting for stochastic GWs and aver- } \\
\text { aging over the source position }\end{array}$ & 0.3190 & 0.3440 & 0.3370 \\
\hline Only averaging over the source position & 0.3182 & 0.3456 & 0.3362 \\
\hline $\begin{array}{l}\text { Relative contribution of stochastic GWs } \\
\text { (absolute value, in \%) }\end{array}$ & 0.3 & 0.5 & 0.2 \\
\hline
\end{tabular}

Table 2. The same as in table 1, but for the inverted mass ordering. The upper row is based on figure 3(b).

that we adopt the following ratio of the fluxes in a source: $(1: 2: 0)_{\mathrm{S}}$.

One can evaluate the effect of stochastic GWs on the measured fluxes in a detector using the data in tables 1 and 2. For this purpose, in the middle rows in tables 1 and 2, we represent the fluxes in a detector in the case when only the averaging over the distance between a source and a detector is made, without accounting for the action of GWs. These values are based on eq. (A.4) and also correspond to $(1: 2: 0)_{\mathrm{S}}$. The neutrino mixing angles for both the normal and inverted mass orderings are taken from ref. [18].

The difference between the upper and middle rows in tables 1 and 2 is the measure of the contribution of stochastic GWs to the fluxes of flavor neutrinos. It is represented in the lower rows in tables 1 and 2. One can see that this contribution is below $1 \%$.

We note that the above analysis is valid if the constraint in eq. (2.6) is fulfilled. To 
get the maximal left hand side in eq. (2.6) we take $\omega=\omega_{\max }=2 \pi f_{\max }, E=10^{2} \mathrm{MeV}$ and $L=1 \mathrm{kpc}$. We obtain that this condition is satisfied for such values.

Now we briefly consider another source of stochastic GWs, like merging binary BHs of stellar masses. We can take that $\alpha=2 / 3$ [27]. The spectral density has the form,

$$
\Omega(f)= \begin{cases}\Omega_{\min }\left(\frac{f}{f_{\min }}\right)^{2 / 3}, & \text { if } f_{\min }<f<f_{\max }, \\ 0, & \text { otherwise }\end{cases}
$$

where $f_{\min }=10^{-5} \mathrm{~Hz}, f_{\max }=10^{2} \mathrm{~Hz}$, and $\Omega_{\min }=10^{-15}[26]$. Unfortunately, it is not possible to express the function $g(t)$ in eq. (2.20) in the explicit form for $\Omega(f)$ in eq. (3.3).

We have solved eq. (2.14) for this case. The value of $\left\langle\rho_{\mathrm{I}}\right\rangle(t)$ turns out to be unchanged for $f_{\min \text { max }}$ and $\Omega_{\min }$ given above for both normal and inverted mass orderings. Thus the evolution of $\left\langle\rho_{\mathrm{I}}\right\rangle(t)$ is similar to that shown in figures $1(\mathrm{~b})$ and $2(\mathrm{~b})$. We have checked $E$ down to $1 \mathrm{MeV}$ and $L$ up to $1 \mathrm{Gpc}$.

Thus, merging BHs with stellar masses as sources stochastic GWs do not result in an effective relaxation of the fluxes of astrophysical neutrinos. Such sources of GWs are more abundant than SMBHs studied above. Nevertheless the typical frequencies of the spectrum of GWs emitted are much higher that in case of SMBHs. It is the main reason why this kind of GWs does not contribute to the relaxation of neutrino fluxes.

\section{Discussion}

In the present work, we have studied the evolution of three mixed flavor neutrinos accounting for their interaction with stochastic GWs with arbitrary spectrum. In section 2, we have derived eqs. (2.14) and (2.15) for the density matrix for flavor neutrinos. This equation generalizes the result of ref. [14], where the $\delta$-correlator of the GW amplitudes was assumed.

In realistic situations, the spectral density of stochastic GWs is a certain function of the frequency in a confined frequency range. It leads to the correlator of amplitudes not necessarily proportional to a $\delta$-function. Thus, the approximation made in ref. [14] is quite rough.

In ref. [14], the estimate of the correlation time $\tau$, or the typical frequency of the spectrum $\tilde{f} \sim \tau^{-1}$, is the main source of the inexactitude in the description of the relaxation of neutrino fluxes. Indeed, $g \sim \tilde{f}^{-3}$ in eq. (2.20) and $\tilde{f}$ is in a quite broad range. Therefore,

slightly changing $\tilde{f}$, we get a significant variation of the relaxation length of the neutrino fluxes.

In the present work, we have avoided this uncertainty. The lower part of the spectrum $\Omega(f)$ turns out to give the main contribution to the relaxation of the fluxes. It can explain much faster relaxation of the fluxes compared to ref. [14].

We have considered the application of our results for the evolution of fluxes of astrophysical neutrinos in section 3. We have studied merging binary BHs as sources of stochastic gravitational waves. We have considered two cases: SMBHs and BHs with stellar masses. In the case of SMBHs, the relaxation distance should be $L \gtrsim 1 \mathrm{kpc}$ for the fluxes to reach their asymptotic values. The relaxation of fluxes is sizable for neutrinos with energies $E \lesssim 10^{2} \mathrm{MeV}$. Thus, the effect of the relaxation of the neutrino fluxes can be important for supernova $(\mathrm{SN})$ neutrinos in our galaxy, with $L_{\mathrm{MW}}=32 \mathrm{kpc}$, since the typical energy of such neutrinos $E_{\mathrm{SN}} \sim 10 \mathrm{MeV}$. 
A strong evidence for the existence of the stochastic GWs background, generated by SMBHs, is reported in ref. [28] recently. The typical frequency of the observed signal is $\sim 10^{-8} \mathrm{~s}^{-1}$. Such a frequency is within the range used in eq. (3.1).

The fluxes of SN neutrinos were reported in ref. [29] to be modified by the mixing between active and hypothetical sterile neutrinos. We have demonstrated that the interaction of active neutrinos with stochastic GWs background with realistic characteristics can also slightly modify the observed SN neutrino fluxes. Perhaps, the predicted effect can be observed by the existing [30] or future [31,32] neutrino telescopes.

Merging BHs with stellar masses are more abundant than coalescing SMBHs. However the lower frequency of their spectra is much higher than that of SMBHs; cf. eqs. (3.1) and (3.3). Thus the effect of the relaxation of neutrino fluxes is smaller in this case. This fact has been confirmed by our numerical simulations.

We have revealed that the neutrino interaction with stochastic GWs results in the relaxation of neutrino fluxes. However, it is not the only random factor affecting the observed fluxes of astrophysical neutrinos. In a realistic situation, a neutrino telescope detects particles emitted by multiple randomly distributed sources.

In section 3 , we have accounted for this factor by considering oscillations in vacuum and averaging the observed fluxes over the distance between a detector and sources. The corresponding fluxes are derived in appendix A; cf. eq. (A.4). Comparing the case when stochastic GWs and the distance averaging are accounted for with the situation when only the distance averaging is made, one can extract the contribution of stochastic GWs to the measured neutrino fluxes; cf. tables 1 and 2. This contribution turns out to be below $1 \%$ level.

Despite the obtained contribution of GWs is small, one can express a restrained hope that a development of experimental techniques will allow one to explore stochastic GWs through a precise measurement of astrophysical neutrino fluxes rather than using direct methods described in ref. [33].

Accounting for the interaction with stochastic GWs and averaging over the positions of randomly distributed neutrino sources, we have obtained that the fluxes at a detector are not equal: $\left(F_{\nu_{e}}: F_{\nu_{e}}: F_{\nu_{e}}\right)_{\oplus} \neq(1: 1: 1)$. We have demonstrated in appendix $\mathrm{A}$ that the situation $\left(F_{\nu_{e}}: F_{\nu_{e}}: F_{\nu_{e}}\right)_{\oplus}=(1: 1: 1)$ is possible only when the initial ratio of fluxes is $(1: 1: 1)_{\mathrm{S}}$. This problem of the flavor content of astrophysical neutrinos was studied in ref. [34]. The fluxes of ultra high energy neutrinos at a detector were found in ref. [34] to depend on the channel of the production of these particles.

In the present work, we have demonstrated that the interaction of astrophysical neutrinos with stochastic GWs can result in the deviation of the flavor ratio in a detector from the value $(1: 1: 1)$. The predicted fluxes are not excluded by the recent observation of ultra high energy astrophysical neutrinos reported in ref. [35]. Plans to improve the sensitivity in the determination of the flavor ratio of astrophysical neutrinos are outlined in ref. [36].

In a realistic situation, one registers the total flux of astrophysical neutrinos with a terrestrial detector, without any information about neutrino sources. Moreover, a specific neutrino source can have a certain energy spectrum. Thus, one has to average the final fluxes over the initial ones accounting for their nontrivial energy spectra. ${ }^{1}$ The same procedure should be made when neutrino oscillations are considered in the presence of stochastic GWs. To evaluate the contribution of GWs, one has to compare these two cases, as it was made

\footnotetext{
${ }^{1}$ In the present work, we adopt the simplest situation of a monocromatic neutrino source.
} 
in section 3. This contribution is expected to be quite small. However, a careful separate analysis is required to get a quantitative result.

In summary, we have studied the evolution of fluxes of astrophysical neutrinos interacting with stochastic GWs having an arbitrary energy spectrum emitted by randomly distributed realistic binary BHs. The consideration of the nontrivial spectrum allowed us to significantly reduce the relaxation distance traveled by a neutrino beam for different flavors to reach the asymptotic values. We could also increase the neutrino energy, for which the relaxation becomes sizable. Now the predicted effect can be potentially observed even for SN neutrinos propagating within our Galaxy.

\section{Acknowledgments}

This work is performed within the government assignment of IZMIRAN.

\section{A Fluxes of neutrinos emitted by random sources}

In this appendix, we derive the expressions for the probabilities to detect a certain flavor of neutrinos produced by multiple, randomly distributed sources accounting for only flavor oscillations in vacuum.

We suppose that a beam of flavor neutrinos is emitted with the probabilities $P_{\lambda}(0)=$ $\left|\nu_{\lambda}(0)\right|^{2}$, where $\lambda=e, \mu, \tau$ and $\left|\nu_{\lambda}(0)\right\rangle$ is the wave function of flavor neutrinos at a source. This neutrino beam is observed in a detector, which is at the distance $L \approx t$ from a source. We suppose that these neutrinos propagate in vacuum.

Using eqs. (2.1) and (2.8), where we keep only $H_{0}$ in $H_{f}$, we get the wave function of flavor neutrinos in a detector,

$$
\left|\nu_{\lambda}(L)\right\rangle=\sum_{a \sigma} U_{\lambda a} U_{\sigma a}^{*} e^{-\mathrm{i} E_{a} L}\left|\nu_{\sigma}(0)\right\rangle,
$$

where $E_{a}=\sqrt{p^{2}+m_{a}^{2}}$ is the energy of the mass eigenstate and $\left(U_{\lambda a}\right)$ are the components of the mixing matrix $U$.

Using eq. (A.1) and ref. [17, pgs. 247-252], we get the probabilities to observe a cetrain flavor $\lambda$ in this neutrino beam,

$$
\begin{aligned}
P_{\lambda}(L)= & P_{\lambda}(0)+2 \sum_{\sigma} P_{\sigma}(0)\left[\sum_{a>b} \operatorname{Im}\left(U_{\lambda a} U_{\lambda b}^{*} U_{\sigma b} U_{\sigma a}^{*}\right) \sin \left(\frac{\Delta m_{a b}^{2}}{2 E} L\right)\right. \\
& \left.-2 \sum_{a>b} \operatorname{Re}\left(U_{\lambda a} U_{\lambda b}^{*} U_{\sigma b} U_{\sigma a}^{*}\right) \sin ^{2}\left(\frac{\Delta m_{a b}^{2}}{4 E} L\right)\right] .
\end{aligned}
$$

To derive eq. (A.2) we take that $\left\langle\nu_{\sigma}(0) \mid \nu_{\kappa}(0)\right\rangle=\delta_{\sigma \kappa} P_{\sigma}(0)$.

Now we suppose that there are multiple neutrino sources with the random distribution with respect to a detector. Such a detector will register a neutrino background averaged over the source position. Therefore we account for the identities,

$$
\left\langle\sin \left(\frac{\Delta m_{a b}^{2}}{2 E} L\right)\right\rangle=0, \quad\left\langle\sin ^{2}\left(\frac{\Delta m_{a b}^{2}}{4 E} L\right)\right\rangle=\frac{1}{2} .
$$


The mechanism of the neutrino emission is supposed to be the same in all the sources, i.e. $P_{\sigma}(0)$ is unchanged in the averaging in eq. (A.3). Finally, we get the averaged probabilities, or the fluxes, of flavor neutrinos in a detector,

$$
\left\langle P_{\lambda}(L)\right\rangle=P_{\lambda}(0)-2 \sum_{\sigma} P_{\sigma}(0) \sum_{a>b} \operatorname{Re}\left(U_{\lambda a} U_{\lambda b}^{*} U_{\sigma b} U_{\sigma a}^{*}\right),
$$

which generalizes the result in ref. [14], which was obtained in the two flavors approximation.

The averaged fluxes in a detector $\left\langle F_{\nu_{\lambda}}\right\rangle_{\oplus} \sim\left\langle P_{\lambda}(L)\right\rangle$ can be in the ratio $(1: 1: 1)_{\oplus}$, suggested in ref. [25], only if the fluxes in a source $\left(F_{\nu_{\lambda}}\right)_{\mathrm{S}} \sim P_{\lambda}(0)$ are equal for any neutrino flavor, i.e. $P_{\lambda}(0)=1 / 3$ for $\lambda=e, \mu \tau$, or $(1: 1: 1)_{\mathrm{S}}$. In general case, including the situation $(1: 2: 0)_{\mathrm{S}}$ in our work, $\left(\left\langle F_{\nu_{e}}\right\rangle:\left\langle F_{\nu_{\mu}}\right\rangle:\left\langle F_{\nu_{\tau}}\right\rangle\right)_{\oplus} \neq(1: 1: 1)$. As results from eq. (A.4), this ratio depends on $\left(F_{\nu_{\lambda}}\right)_{\mathrm{S}}$, or $P_{\lambda}(0)$, and the mixing angles.

\section{References}

[1] The Borexino Collaboration, M. Agostini et al., Improved measurement of ${ }^{8} \mathrm{~B}$ solar neutrinos with $1.5 \mathrm{kt} \cdot y$ of Borexino exposure, Phys. Rev. D 101 (2020) 062001 [arXiv:1709.00756].

[2] M. Fukugita and T. Yanagida, Physics of Neutrinos and Applications to Astrophysics, Springer, Berlin Germany (2003).

[3] D. V. Ahluwalia and C. Burgard, Gravitationally induced quantum mechanical phases and neutrino oscillations in astrophysical environments, Gen. Rel. Grav. 28 (1996) 1161 [gr-qc/9603008].

[4] G. G. Luciano and L. Petruzziello, Testing gravity with neutrinos: From classical to quantum regime, to be published in Int. J. Mod. Phys. D [arXiv:2007.08664].

[5] N. Fornengo, C. Giunti, C. W. Kim, and J. Song, Gravitational effects on the neutrino oscillation, Phys. Rev. D 56 (1997) 1895 [hep-ph/9611231].

[6] LIGO Scientific Collaboration and Virgo Collaboration, B. P. Abbott et al., Observation of gravitational waves from a binary black hole merger, Phys. Rev. Lett. 116 (2016) 061102 [arXiv:1602.03837].

[7] LIGO Scientific Collaboration and Virgo Collaboration, B. P. Abbott et al., GWTC-1: A gravitational-wave transient catalog of compact binary mergers observed by LIGO and Virgo during the first and second observing runs, Phys. Rev. X 9 (2019) 031040 [arXiv:1811.12907].

[8] P. Mészéros, D. B. Fox, C. Hanna, and K. Murase, Multi-messenger astrophysics, Nature Rev. Phys. 1 (2019) 585 [arXiv:1906.10212].

[9] IceCube Collaboration, M. G. Aartsen et al., IceCube search for neutrinos coincident with compact binary mergers from LIGO-Virgo's first gravitational-wave transient catalog, Astrophys. J. Lett. 898 (2020) L10 [arXiv:2004.02910].

[10] M. Dvornikov, Neutrino spin oscillations in gravitational fields, Int. J. Mod. Phys. D 15 (2006) 1017 [hep-ph/0601095].

[11] M. Dvornikov, Neutrino spin oscillations in matter under the influence of gravitational and electromagnetic fields, JCAP 06 (2013) 015 [arXiv:1306.2659].

[12] M. Dvornikov, Spin oscillations of neutrinos scattered off a rotating black hole, Eur. Phys. J. C 80 (2020) 474 [arXiv:2006.01636].

[13] M. Dvornikov, Neutrino spin oscillations in external fields in curved spacetime, Phys. Rev. D 99 (2019) 116021 [arXiv:1902.11285]. 
[14] M. Dvornikov, Neutrino flavor oscillations in stochastic gravitational waves, Phys. Rev. D 100 (2019) 096014 [arXiv:1906.06167].

[15] Y. N. Obukhov, A. J. Silenko, and O. V. Teryaev, General treatment of quantum and classical spinning particles in external fields, Phys. Rev. D 96 (2017) 105005 [arXiv:1708.05601].

[16] G. Koutsoumbas and D. Metaxas, Neutrino oscillations in gravitational and cosmological backgrounds, Gen. Rel. Grav. 52 (2020) 102 [arXiv:1909.02735].

[17] C. Giunti an C. W. Kim, Fundamentals of Neutrino Physics and Astrophysics, Oxford University Press, Oxford U.K. (2007).

[18] P. F. de Salas, D. V. Forero, S. Gariazzo, P. Martínez-Miravé, O. Mena, C. A. Ternes, M. Tórtola, and J. W. F. Valle, 2020 Global reassessment of the neutrino oscillation picture [arXiv:2006.11237].

[19] P. Hoyng, Relativistic Astrophysics and Cosmology: A Primer Springer, Berlin Germany (2006), pgs. 133-136.

[20] N. J. Popławski, A Michelson interferometer in the field of a plane gravitational wave, J. Math. Phys. 47 (2006) 072501 [gr-qc/0503066].

[21] F. N. Loreti and A. B. Balantekin, Neutrino oscillations in noisy media, Phys. Rev. D 50 (1994) 4762 [nucl-th/9406003].

[22] S. Weinberg, The Quantum Theory of Fields. Vol. I. Foundations, Cambridge University Press, Cambridge U.S.A. (1996), pgs. 142-143.

[23] G. A. Korn and T. M. Korn, Mathematical Handbook for Scientists and Engineers: Definitions, Theorems and Formulas for Reference and Review, McGraw-Hill, New York U.S.A. (1968), 2nd ed.

[24] N. Christensen, Stochastic gravitational wave backgrounds, Rep. Prog. Phys. 82 (2019) 016903 [arXiv:1811.08797].

[25] J. G. Learned and S. Pakvasa, Detecting $\nu_{\tau}$ oscillations at PeV energies, Astropart. Phys. 3 (1995) 267 [hep-ph/9408296].

[26] P. A. Rosado, Gravitational wave background from binary systems, Phys. Rev. D 84 (2011) 084004 [arXiv:1106.5795].

[27] LIGO Scientific and Virgo Collaboration, B. P. Abbott et al., Search for the isotropic stochastic background using data from Advanced LIGO's second observing run, Phys. Rev. D 100 (2019) 061101 [arXiv:1903.02886].

[28] NANOGrav Collaboration, Z. Arzoumanian et al., The NANOGrav 12.5-year data set: Search for an isotropic stochastic gravitational-wave background, submitted to Astrophys. J. Lett. [arXiv:2009.04496].

[29] P. Keränen, J. Maalampi, M. Myyryläinen, and J. Riittinen, Sterile neutrino signals from supernovae, Phys. Rev. D 76 (2007) 125026 [arXiv:0708.3337].

[30] V. Kulikovskiy, ANTARES and KMBNeT programs for the supernova neutrino detection, Proc. Int. Astron. Union 12 (2017) 339.

[31] J. Migenda, Supernova burst observations with DUNE [arXiv:1804.01877].

[32] V. Fischer, Theia: A multi-purpose water-based liquid scintillator detector [arXiv:1809.05987].

[33] J. D. Romano and N. J. Cornish, Detection methods for stochastic gravitational-wave backgrounds: A unified treatment, Living Rev. Rel. 20 (2017) 2 [arXiv:1608.06889].

[34] H. Nunokawa, B. Panes, and R. Zukanovich Funchal, How unequal fluxes of high energy astrophysical neutrinos and antineutrinos can fake new physics, JCAP 10 (2016) 036 [arXiv:1604.08595]. 
[35] IceCube Collaboration, M. G. Aartsen et al., Flavor ratio of astrophysical neutrinos above 35 TeV in IceCube, Phys. Rev. Lett. 114 (2015) 171102 [arXiv:1502.03376].

[36] IceCube Collaboration, M. G. Aartsen et al., IceCube-Gen2: The window to the extreme universe [arXiv:2008.04323]. 